\title{
Systemic Colchicine Inhibits Goldfish Optic Nerve Regeneration ${ }^{1}$
}

\author{
Roger E. Davis, Barbara E. Schlumpf, and Paul D. KLinger \\ University of Michigan Mental Health Research Institute and \\ Neuroscience Laboratory, Ann Arbor, Michigan 48109
}

Received August 22, 1983; accepted October 25, 1983

\begin{abstract}
Systemic Colchicine Inhibits Goldfish Optic Nerve Regeneration. DavIS, R. E., SCHLUMPF, B. E., AND KuINGER, P. D. (1984). Toxicol. Appl. Pharmacol. 73, 268-274. Experiments were carried out to further investigate the regenerating goldfish optic nerve as a preparation for screening drugs or environmental toxins for adverse effects on neuronal circuit development. Regeneration was induced by unilateral retrobulbar optic nerve crush, and the opposite eye was kept intact. The time to recovery of vision was measured, as an index of regeneration and neurotoxicity, by an improved behavioral technique. The visual stimulus was changed to eliminate extraretinal photoresponding and to permit testing for vision with the right or left eye independently in a trial. Visual recovery occurred within 14 to 25 days. Colchicine, a potent inhibitor of microtubules and axonal transport, was administered semiweekly by ip injection, as in earlier experiments, to study the efficacy of the protocol. The drug resulted in an inhibition of regeneration at doses up to $0.2 \mu \mathrm{g} / \mathrm{g}$ body wt which did not impair responding with the control eye. Administration of $\beta$-lumicolchicine, a photoisomer of colchicine that is a weak inhibitor of microtubules or axonal transport, up to $2.0 \mu \mathrm{g} / \mathrm{g}$ body wt, had no effect on regeneration or maintenance of visual responding. The results support the thesis that regenerating circuits are more sensitive indicators of neurotoxicity than are established circuits and confirm that the regenerating optic nerve can be used to screen molecules that may impair neuronal circuit development in vivo and to measure their relative potency.
\end{abstract}

Previous experiments by our laboratory showed that the regenerating goldfish optic nerve may be a promising preparation for screening molecules for adverse effects on neuronal circuit development in vivo (Davis and Benloucif, 1981). A protocol was investigated in which regeneration is induced by crushing the optic nerve within the orbit near the eyeball (Attardi and Sperry, 1963: Grafstein and McQuarrie, 1978; Springer and Agranoff, 1977). Neurotoxicity affecting axonal regeneration or the recovery of visual function in the retinal ganglion cells is assessed by measuring the time to recovery of vision. Visual recovery time is measured by a classical

\footnotetext{
' Supported by the U.S. Environmental Protection Agency under Grant 808064-02.
}

conditioning technique in which a branchial suppression response (SR) to a light conditioned stimulus (CS) serves as the response index of vision. The efficacy of the protocol was examined with the alkaloid neurotoxins, colchicine and vincristine sulfate (Ochs, 1974; Banks and Till, 1975; Dahlstrom et al., 1975; Hanson and Edström, 1978), which were administered intraperitoneally twice weekly for 3 weeks following optic nerve crush or a sham operation. Colchicine and vincristine each inhibited recovery of the SR in optic nerve crush fish at systemic concentrations that did not impair maintenance of response in sham-operated fish which indicates that the behavioral protocol can detect an inhibition of optic nerve regeneration produced by systemically administered drugs. However, the study was 
complicated by the finding that the SR could be evoked by extraretinal, as well as retinal, cues produced by the CS (Davis and Benloucif, 1981). Because extraretinal SRs might produce errors in assessing the time to recovery of vision, the protocol was revised (Davis and Schlumpf, 1983).

The present experiments were carried out to reassess the toxicity of colchicine by the revised behavioral assay. In the original protocol, one eye was removed from every fish prior to SR conditioning and the CS consisted of a change-of-luminance CS, that is "on-off," that was produced by turning on a bright light in darkness. The revised protocol employs a moving-spot-of-light CS which avoids the problem of extraretinal photostimulation (Davis and Schlumpf, 1983). The moving-spot CS is presented monocularly so that one eye can be used as the experimental, to detect drug effects on recovery of vision, and the other as a control, to detect effects of maintenance of response. Treatments that inhibit visual recovery after optic nerve crush but do not inhibit maintenance of response with the control eye can be inferred to inhibit optic axon regeneration.

\section{METHODS}

Fish. Goldfish (Carassius auratus L.), 8 to $12 \mathrm{~g}$, obtained from Ozark Fisheries, Stoutland, Missouri, were kept in large tanks at 26 to $28^{\circ} \mathrm{C}$ for several weeks prior to being placed in individual home tanks at $30^{\circ} \mathrm{C}$. Fish were fed Tetramin conditioning flake food once or twice daily. The daily photoperiod was $16: 8$ hr L:D with fluorescent light augmented by diffuse natural light. The tank water was a mixture of distilled and charcoal filtered, softened well water; the conductivity was $450 \pm 50 \mathrm{mmho} / \mathrm{cm}$ and the $\mathrm{pH}$ was 7.0-7.5. Experiments were conducted during January through May.

Surgery. To crush the optic nerve, fish were anesthetized by immersion in $0.04 \%$ trimethanemethylsulfonate (Sigma) in tank water buffered with Tris fish buffer (Sigma) to $\mathrm{pH}$ 6.5-7.5, wrapped in a moist paper towel, and placed on the stage of a microscope. The eyeball was rotated ventrally and the nerve was exposed through a slit that was cut in the conjunctiva dorsal to the eye. The nerve was crushed by pinching it twice with a Dumont No. 7 forcep. The anesthetized fish was returned to the home tank to recover.
Experimental drugs. Colchicine and $\beta$-lumicolchicine (Sigma) were administered ip by a 30-gauge needle and a 100- $\mu$ l Hamilton syringe (Davis and Benloucif, 1981). Colchicine was delivered in $0.15 \mathrm{M} \mathrm{NaCl}$ and lumicolchicine in PET-80 which consisted of 3\% ethanol, 3\% Tween-80, and propylene glycol. The drug was dissolved in the ethanol and Tween-80 at approximately $60^{\circ} \mathrm{C}$ prior to dilution with propylene glycol. The volume of each injection was $1 \mu \mathrm{l} / \mathrm{g}$ body wt.

Conditioning tank and stimuli. Fish were conditioned individually in three glass tanks which were $15 \mathrm{~cm}$ wide, $15 \mathrm{~cm}$ deep, and $30 \mathrm{~cm}$ long and filled with $8 \mathrm{~cm}$ of water heated to $30 \pm 0.5^{\circ} \mathrm{C}$. The fish was restrained in a holder $5 \mathrm{~cm}$ beneath the surface of the water in the center of the tank and $45 \mathrm{~cm}$ beneath two 20-W, cool-white fluorescent lamps which were continuously illuminated, and the fish's branchial movements were detected by a thermistor. The CS consisted of the flickering movement of a spot of red light, which was produced by alternately illuminating two light-emitting diodes (LEDs, 4-mm diam; General Instrument, MV 5732) that were centered one above the other $1 \mathrm{~cm}$ apart. Similar pairs of LEDs were submerged in the water opposite each eye. The upper LED was approximately $2.5 \mathrm{~cm}$ from the eye and $1 \mathrm{~cm}$ below its horizontal axis and the anglc of vicw subtended by the spot of light was $10^{\circ}$. The lower LED was kept lit during the intertrial interval. During the CS interval, the upper and lower LED were illuminated alternately every 250 msec. This procedure produced movement of the spot of light through an angle of $26^{\circ}$. The unconditioned stimulus (US) was a 0.25 -sec pulse from 7- to 8-mA (RMS), 60$\mathrm{Hz}$ constant current that was passed between two steel electrodes on opposite sides of the fish holder (Davis, 1981; Davis and Schlumpf, 1983).

Preliminary conditioning. Fish were administered two or three semiweekly sessions of conditioning trials. Each session consisted of twenty 2-sec CS-US trials, followed by four 5 -sec CS-US test trials. The CS was turned on for 2 or $5 \mathrm{sec}$ and the US was presented at the instant that the CS was terminated. The 2-sec trials were initiated automatically by a microprocessor at random intervals of 0.5 to $3 \mathrm{~min}$ in duration and the fish's response to the CS was not recorded. The CS was presented to the right (R) or left (L) eye in pseudorandom order. The SR to the CS was recorded in the 5-sec trials, which were initiated by the investigator at times when the fish's branchial movements were regular, and the branchial beat rate was measured by a tachograph method (Davis and Schlumpf, 1983). A microprocessor counted the number of branchial beats during the 5-sec interval (B) of the test trial, and the number during the 5-sec interval (A) preceding the onset of the trial and calculated the percentage change in the rate in interval B relative to the rate in A [ $100 \times(1$ $-B / A)]$ which is noted as the SR. A SR of greater than $30 \%$ was accepted as a response indicative of vision. Fish that responded in R-and L-trials were retained as potential subjects (Davis and Schlumpf, 1983). 
Preoperative test sessions. Within a week prior to an experiment, potential subjects received a test session consisting of four 2-sec trials (order: RLRL) followed by four 5 -sec test trials (RLRL). The occurrence of false-positive responses was assessed in blank trials administered prior to each test trial. Fish that responded to the CS (SR greater than $30 \%$ ) in each of the four test trials and showed no false positives were accepted as subjects.

Assay protocol and data analysis. Subjects received right optic nerve crush and ip administration of either colchicine, lumicolchicine, saline, or PET-80. The left optic nerve was kept intact. The drugs were administered the day before surgery and following each postaxotomy test session. The day of optic nerve crush was designated as Day 0 , and test sessions were given twice weekly beginning at 4 days postaxotomy (DPA). The procedure in each session was the same as in the preoperative test session.

The data for each session were reduced by computing the average SR (percentage suppression) in R- or L-trials for each fish. The effects of the various doses of colchicine on the mean SR were analyzed initially in an ANOVA for one grouping factor, Dose, with two within factors, Day $(4,7, \ldots$ ) and Eye ( $R, L)$. Between-group differences in recovery of vision were further evaluated by the ratio of the average $S R$ in R-trials to the average SR in L-trials, which is designated as the Sightedness $(S)$ score. The mean $S$ scores of the various groups were contrasted in an ANOVA for one grouping factor, Dose, with one within factor, Day. BMDP statistical software was used (Dixon $e t$ al., 1981).

The $50 \%$ effective dose (ED50) was defined as the dose that is estimated to produce a mean $S$ score of 0.5 on 21 DPA. The 21-day time point was taken as an approximation of the time following axotomy when the mean $S$ score for control fish approaches unity. The 21-day ED50 and $95 \%$ confidence limits were derived from the regression of the mean $S$ score against the $\log$ of the dose +1 .

\section{RESULTS}

Colchicine inhibited visual recovery increasingly with the dose up to $0.2 \mu \mathrm{g} / \mathrm{g}$ body wt which was the highest dose tested (Fig. 1). The ANOVA on the mean $S$ score for the saline and the colchicine groups revealed significant Dose $[F(4,32)=5.12, p<0.01]$ and Day $[F(6,192)=45.53, p<0.01]$ effects and a Dose $\times$ Day interaction $[F(24,192)=3.93$, $p<0.01$ ]. Three fish receiving $0.2 \mu \mathrm{g} / \mathrm{g}$ died, one at $11 \mathrm{DPA}$ and two at $18 \mathrm{DPA}$, and their data were omitted. The 21-day ED50 was 0.08 $(0.06$ to 0.10$) \mu \mathrm{g} / \mathrm{g}$.

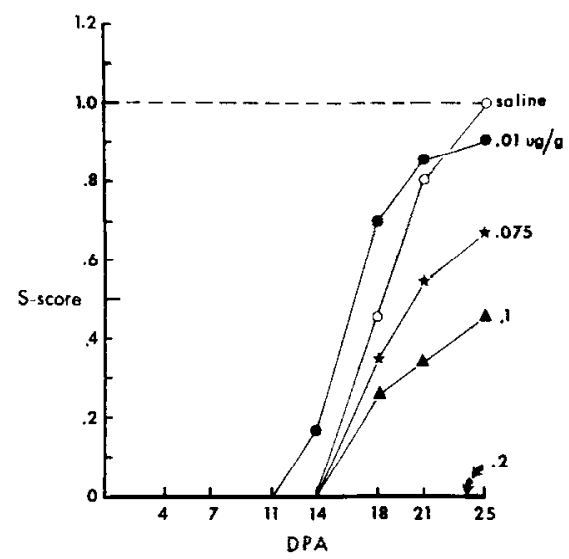

FIG. 1. Effects of ip administered colchicine on recovery of R-ye sightedness ( $S$ score) following R-optic nerve crush, Mean $S$ scores of less than 0.05 were not plotted.

Whether colchicine impaired maintenance or only recovery of response was determined by examining the effects of Dose or Day on the mean SR in L- or R-trials (Eye factor). The primary ANOVA showed significant Dose $[F(4,32)=2.81, p<0.05]$, Eye $[F(1,32)$ $=833.34, p<0.01]$, and Day $[F(6,192)$ $=35.08, p<0.01]$ effects and Dose $\times$ Eye $\times$ Day interaction $[F(24,192)=3.59, p$ $<0.01]$. An ANOVA on the R-trial data showed significant Dose $[F(4,32)=4.92$, $p<0.01]$ and Day $[F(6,192)=45.52, p$ $<0.01]$ effects and Dose $\times$ Day interaction $[F(24,192)=3.79, p<0.01]$. In contrast, the mean SR in L-trials was unaffected by Dose $[F(4,32)=1.24 p>0.3]$ or Day $[F(6,192)$ $=1.62, p>0.3]$. Thus, there was no evidence of an effect on maintenance of response to the CS.

Lumicolchicine effects. Fish administered $\beta$-lumicolchicine, 0.2 or $2.0 \mu \mathrm{g} / \mathrm{g}$ body $\mathrm{wt}$, saline, or PET-80 without a drug showed no significant differences in recovery of R-eye sightedness $[F(18,180)=1.40, p>0.1]$ (Fig. 2 ). The groups showed uniformly high levels of response in L-trials and the recovery of the mean SR in R-trials closely paralleled the mean $S$ score. ANOVAs on the mean SR for the four groups revealed significant Eye $[F(1,29)=526.74, \quad p<0.01]$ and Day $[F(6,174)=89.69, p<0.01]$ effects and an 


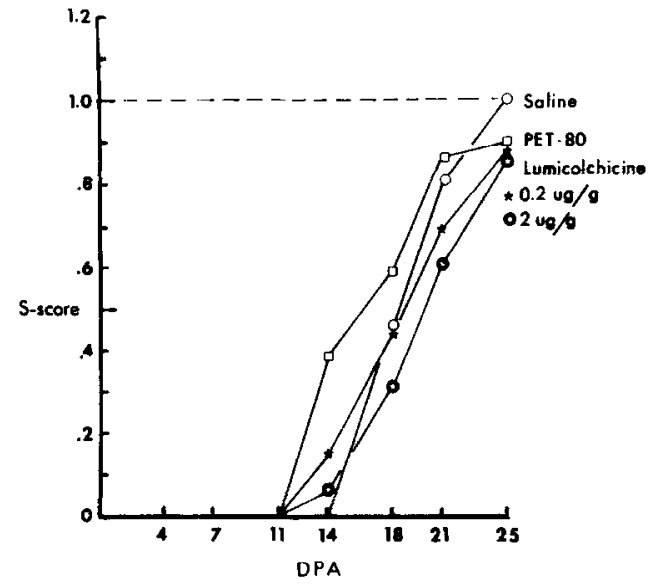

FIG. 2. Effects of ip administered $\beta$-lumicolchicine or saline or PET-80 on recovery of R-eye sightedness ( $S$ score) following R-optic nerve crush. Mean $S$ scores of less than 0.05 were not plotted.

Eye $\times$ Day interaction $[F(6,174)=119.85, p$ $<0.01]$. Day affected the mean SR in R-trials $[F(6,180)=115.5, p<0.01]$ but not L-trials $[F(6,180)=0.53, p>0.05]$.

\section{DISCUSSION}

\section{Improved Measure of Drug Effect on Optic Nerve Regeneration}

This investigation confirms and extends the earlier report (Davis and Benloucif, 1981) that the time to recovery of a branchial SR to a conditioned visual CS can be used to measure the effects of systemically administered molecules on optic nerve regeneration. The revised protocol detected effects of colchicine at doses lower than the original as can be seen in Fig. 3. The key revision may be in the CS, namely, changing the on-off CS, which might have evoked extraretinal SRs during the postaxotomy period prior to visual recovery, to the more refined moving-spot CS that does not evoke the SR extraretinally (Davis and Schlumpf, 1983; unpublished data). Extraretinal SRs would result in an underestimation of visual recovery time and, thereby, the toxicity of colchicine to regenerating optic axons.
Other revisions that were made which might produce more accurate measurements of visual recovery time were improved water quality and more uniform temperatures in the home tank and the experimental tank, a more objective method of measuring the branchial SR, and the use of a within-fish, rather than between-fish, experimental design to dissociate drug effects on recovery and maintenance of response. The difference in the dose-response curves obtained with the original and the revised protocols does not appear to be a result of using the mean $S$ score, instead of the percentage-of-fish responding, as the measure of visual recovery (Fig. 3).

\section{Mode of Action of Colchicine}

Colchicine is a potent inhibitor of microtubules and axonal transport (Ochs, 1974; Paulson and McClure, 1975; Hanson and Ed-

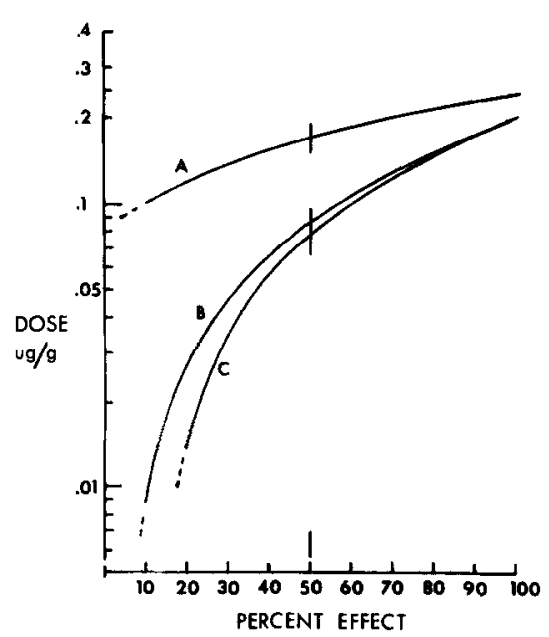

FIG. 3. Colchicine dose-response curves for the original and revised protocols. Curve $A$ is the regression of the dose against the percentage of fish responding (SR $>30 \%$ ) at 17 DPA in the original protocol. Curves $B$ and $C$ show the regression of the dose against, respectively, the percentage of fish responding or the mean $S$ score $(\times 100)$ on $21 \mathrm{DPA}$ in the revised protocol. The vertical intersects denote the ED50 which was calculated to be $0.17(0.15$ to 0.18$) \mu \mathrm{g} / \mathrm{g}$ body wt for $\mathrm{A}, 0.09(0.06$ to 0.11$) \mu \mathrm{g} / \mathrm{g}$ for $\mathrm{B}$, and $0.08(0.05$ to 0.10$) \mu \mathrm{g} / \mathrm{g}$ for $\mathrm{C}$. 
ström, 1978) which are essential for neuronal growth and development (Grafstein, 1975; Grafstein and Foreman, 1980) and for the maintenance of function in established neuronal circuits (Perisic and Cuenod, 1972). The inhibitory effects of colchicine and other alkaloid antimitotic drugs have been the main evidence that microtubules participate in axonal transport. These alkaloids have a high affinity for tubulin, the subunit protein of microtubules, which suggests that they may compete with endogenous inhibitors of microtubule polymerization (Sherline et al., 1979), but they also bind to other axoplasmic proteins and membranes and are thus not specific inhibitors of microtubules (Feit and Barondes, 1970).

Colchicine taken up into retinal ganglion cells of the experimental eye could inhibit axonal outgrowth to brain visual centers or the formation of synaptic connections between the incoming optic axons and their targets. Axonal sprouting (Hoffman, 1952), peripheral nerve fiber regeneration (Mellick et al., 1974), and neurite outgrowth in vitro (Daniels, 1972) are reported to be inhibited by colchicine. Experiments in pigeons showed that synaptic transmission in optic axons was decreased following an intraocular injection of colchicine that inhibited axonal transport (Perisic and Cuenod, 1972), but effects on vision were not reported. Axonal transport in trout optic nerve was inhibited following a single intraocular administration of approximately 1 but not 0.5 $\mu \mathrm{g} / \mathrm{g}$ body wt colchicine (Schönhartung et al., 1977).

Lumicolchicine is a photoisomer of colchicine that binds to various axoplasmic proteins but, in contrast to colchicine, has a very low affinity for tubulin (Banks and Till, 1975) and is a correspondingly weak inhibitor of microtubule assembly or axonal transport (Dahlstrom et al., 1975; Hanson and Edström, 1978). Systemic concentrations up to $2 \mu \mathrm{g} / \mathrm{g}$ body wt lumicolchicine, which is 25 times the ED50 for colchicine, had no effect on visual recovery. The ineffectiveness of lumicolchicine suggests that systemic colchicine inhibits visual recovery, at least in part, by inhibiting microtubules or axonal transport.

\section{CS-Related Differences in Visual Recovery Time}

Our experiments provide the first evidence that the time to recovery of a response, in this case a conditioned branchial SR, varies with the properties of the visual stimulus. Other laboratories have demonstrated that recovery time varies between protocols with different responses and stimuli. For example a wholebody photic startle response to sudden overhead illumination (Edwards et al., 1981) reappears in 1 to 2 weeks; an unconditioned branchial SR to an overhead shadow (Springer and Agranoff, 1977) and a conditioned branchial SR to a relatively bright on-off CS (Davis and Benloucif, 1981) reappear within 2 to 3 weeks; and a whole-body pursuit response to a moving visual field is restored several weeks later (Springer and Agranoff, 1977).

Control nerve crush fish showed initial recovery of the branchial SR to the on-off CS of the original protocol within 17 DPA and to the moving-spot CS within 25 DPA. The increased time to recovery suggests that the neuronal regeneration processes involved may differ. Detection of the moving-spot CS might require a more extensive restoration of the retinotectal system and, thereby, a longer period of recovery (Springer and Agranoff, 1977) or the completion of recovery processes within retinal ganglion cells (Edwards et al,, 1981) or within postsynaptic neurons that are not required for detection of the on-off CS. The conclusion that the time to recovery of the SR is stimulus dependent is supported by a pilot study with a different stimulus. We had planned to use a moving-ring CS which employed "linear" instead of "spot" light-emitting diodes (Davis and Schlumpf, 1983), but recovery times were 7 to 10 days longer than for the moving-spot CS, and the latter was adopted for experimental efficiency. 


\section{Dissociation of Regeneration and Maintenance Effects}

The fact that colchicine inhibits recovery of response at systemic doses below those that inhibit maintenance of response in the same individual supports the thesis that regenerating or developing neuronal circuits may be more effective indicators of neurotoxicity than are mature or established circuits (Davis and Benloucif, 1981). Systemic doses of colchicine that inhibit optic nerve regeneration are presumably toxic to normal retinal and other neurons and to many other types of cells throughout the body. Several fish receiving the $0.2 \mu \mathrm{g} / \mathrm{g}$ dose died during experiments and increasing the dose to $0.3 \mu \mathrm{g} / \mathrm{g}$ is lethal to most individuals in 21 days.

The possibility that colchicine was unevenly distributed in the experimental and control optic systems needs to be considered. Since the blood-brain barrier may normally exclude most systemic colchicine (Bennett and Alberti, 1981) and crushing the optic nerve ruptures the barrier (Kiernan and Contestabile, 1980), there may be greater uptake in the experimental as opposed to the control optic system. Drugs taken up in the experimental optic nerve would presumably diffuse into other brain structures including visual centers that mediate control eye responding. While additional experiments are needed to clarify whether the blood-brain barricr could be rcsponsible for the greater effect of colchicine on regenerating, as opposed to normal optic axons, the procedure of testing for regeneration and maintenance effects in the same individual is an improvement over the original between-fish design.

In conclusion, our results indicate that the time to visual recovery following optic nerve crush can be used to screen molecules for adverse effects on neuronal circuit development. The assay has several important features. Drug effects are assessed at the cellular level in a well-known and anatomically localized population of neurons, the retinal ganglion cells, in vivo. Regenerating optic axons may be more sensitive indicators of neurotoxicity than are mature axons and, therein, better suited for detecting sublethal toxicity. The assay should be sensitive to molecules that inhibit (or promote) neuronal metabolism, biosynthesis, axonal transport, or any aspect of neuronal fiber development or synapse formation. Optic nerve regeneration is a pathological preparation but it seems reasonable to assume that it includes many cellular processes that occur during normal neuronal circuit development. Thus, evidence that a drug inhibits optic nerve regeneration would strongly imply that it may be injurious to normal neurons.

\section{REFERENCES}

ATtardi, D. G., AND SPERry, R. W. (1963). Preferential selection of central pathways by regenerating optic fibers. Exp. Neurol. 7, 46-64.

BANKS, P., AND TILL, R. (1975). A correlation between the effects of anti-mitotic drugs on microtubule assembly in vitro and the inhibition of axonal transport in noradrenergic neurones. J. Physiol. 252, 283-294.

BENNETT, E. L., AND ALBERTI, M. H. (1981). Uptake of $\left[{ }^{3} \mathrm{H}\right]$ colchicine into brain and liver of mouse, rat, and chick. Pharmacol. Biochem. Behav. 14, 863-869.

Dahlstrom, A., Heiwall, P. O., and Larsson, P. A. (1975). Comparison between the effect of colchicine and lumicolchicine on axonal transport in rat motor neurons. J. Neurol. Trans. 37, 305-311.

DANIELS, M. P. (1972). Colchicine inhibition of nerve fiber formation in vitro. J. Cell Biol. 53, 164-176.

DAVIS, R. E, (1981). A method of restraining goldfish during conditioning of electric-shock-induced suppression of breathing movements. Behav. Res. Methods Instrument. 13, 650-652.

DAVIS, R. E., AND BENLOUCIF, S. (1981). Behavioral investigation of neurotoxicity: The effects of colchicine, lumicolchicine and vincristine sulfate on goldfish optic nerve regeneration. Neurotoxicology 2, 419-430.

DAVIS, R. E., AND SCHLUMPF, B. E. (1983). Circumvention of extraretinal pholoresponses in assessing the recovery of vision in goldfish following optic nerve crush. Behav. Brain Res. 7, 65-79.

Dixon, W. J., Brown, M. B., Engelman, L., Frane, J. W., Hill, M. A., JENNRICH, R. I., AND TOPOREK, J. D. (1981). BMDP Statistical Software, pp. 347-349. Univ. of California Press, Berkeley.

Edwards, D. L., Alpert, R. M., and Grafstein, B. (1981). Recovery of vision in regeneration of goldfish 
optic axons: Enhancement of axonal outgrowth by a conditioning lesion. Exp. Neurol. 72, 672-687.

FEIT, H., AND BARONDES, S. H. (1970). Colchicine-binding activity in particulate fractions of mouse brain. $J$. Neurochem. 17, 1355-1364.

GRAFSTEIN, B. (1975). The nerve-cell body response to axotomy. Exp. Neurol. 48, 32-51.

GRAFSTEIN, B., AND FORMAN, D. S. (1980). Intracellular transport in neurons. Physiol. Rev. 60, 1167-1283.

GRAFSTEIN, B., AND MCQUARRIE, I. G. (1978). Role of the nerve cell body in axonal regeneration. In Neuronal Plasticity (C. W. Cotman, ed.), pp. 155-195. Raven Press, New York.

HANSON, M., AND EDSTRÖM, A. (1978). Mitosis inhibitors and axonal transport. Int. Rev. Cytol. Suppl. 7, 373402.

HoFFMAN, H. (1952). Acceleration and retardation of the process of axonal-sprouting in partially denervated muscles. Aust. J. Exp. Med. Sci. 30, 541-554.

KiERNAN, J. A., AND CONTESTABILE, A. (1980). Vascular permeability associated with axonal regeneration in the optic system of the goldfish. Acta Neuropathol. (Berlin) 51, 39-45.

Mellick, R., KIRKBy, R., SMITH, A. T., AND RATNA-
VALE, G. (1974). Colchicine and the peripheral nerve. Proc. Aust. Assoc. Neurol. 11, 69-76.

OCHS, S. (1974). Systems of material transport in nerve fibers (axoplasmic transport) related to nerve function and trophic control. Ann. New York Acad. Sci. 228, 202-223.

Paulson, J. C., And MCClure, W. O. (1975). Inhibition of axoplasmic transport by colchicine, podophyllotoxin, and vinblastine: An effect on microtubules. Ann. New York Acad. Sci. 253, 517-527.

Perisic, M., AND Cuenod, M. (1972). Synaptic transmission depressed by colchicine blockade of axoplasmic flow. Science 175, 1140-1142.

SChÖNhartung, M., Breer, H., RahmanN, H., GuNTER, S., AND RAENER, H. (1977). Colchicine, a novel inhibitor of fast axonal transport without tubulin binding properties. Cytobiologie 16, 106-117.

Sherline, P., Leung, J. T., AND KIPNIS, D. M. (1979). Binding of colchicine to purified microtubule protein. J. Biol. Chem. 250, 5481-5486.

SPRINGER, A. D., AND AGRANOFF, B. W. (1977). Effect of temperature on rate of goldfish optic nerve regeneration: A radioautographic and behavioral study. Brain Res. 128, 405-415. 